\title{
OPEN Absence of relevant QT interval prolongation in not critically ill COVID-19 patients
}

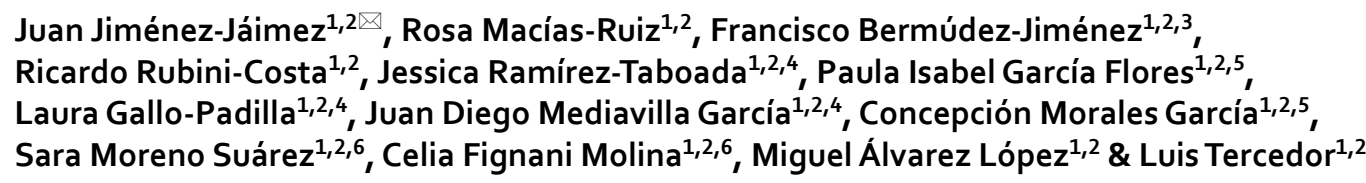

SARS-CoV-2 is a rapidly evolving pandemic causing great morbimortality. Medical therapy with hydroxicloroquine, azitromycin and protease inhibitors is being empirically used, with reported data of QTc interval prolongation. Our aim is to assess QT interval behaviour in a not critically ill and not monitored cohort of patients. We evaluated admitted and ambulatory patients with COVID-19 patients with 12 lead electrocardiogram at $48 \mathrm{~h}$ after treatment initiation. Other clinical and analytical variables were collected. Statistical analysis was performed to assess the magnitude of the QT interval prolongation under treatment and to identify clinical, analytical and electrocardiographic risk markers of QT prolongation independent predictors. We included 219 patients (mean age of $63.6 \pm 17.4$ years, $48.9 \%$ were women and $16.4 \%$ were outpatients. The median baseline QTc was $416 \mathrm{~ms}$ (IQR 404-433), and after treatment QTc was prolonged to $423 \mathrm{~ms}(405-438)(P<0.001)$, with an average increase of $1.8 \%$. Most of the patients presented a normal QTc under treatment, with only 31 cases (14.1\%) showing a QTc interval > $460 \mathrm{~ms}$, and just one case with QTc > $500 \mathrm{~ms}$. Advanced age, longer QTc basal at the basal ECG and lower potassium levels were independent predictors of QTc interval prolongation. Ambulatory and not critically ill patients with COVID-19 treated with hydroxychloroquine, azithromycin and/or antiretrovirals develop a significant, but not relevant, QT interval prolongation.

Since late 2019, a new coronavirus is responsible for the Severe Acute Respiratory Syndrome Coronavirus 2 (SARS-CoV-2) causing a worldwide pandemic ${ }^{1}$. It has spread rapidly throughout the world with a high mortality rate, especially among older adults with cardiovascular disease ${ }^{2}$. There is currently no specific preventive or therapeutic strategy approved by the European Medicines Agency (EMA) or the Food and Drug Administration (FDA), so an empiric approach with conventional antimalarial, antibiotic or antiviral drugs are being evaluated for COVID-19. Some of them have been proven to have in-vitro antiviral effects against SARS-CoV-2. Particularly hydroxychloroquine (HCQ) seems to limit the virus entry likely through inhibition of angiotensinconverting enzyme 2 (ACE2) receptors ${ }^{3}$. Recently, one study has suggested that the concomitant use of azithromycin in combination with HCQ is associated with a more rapid resolution of virus detection than HCQ alone $e^{4}$. Finally, lopinavir/ritonavir is a protease inhibitor largely used in HIV infection, and has in-vitro activity against SARS-CoV-2 ${ }^{5}$. While the optimal strategy to treatment of COVID-19 is uncertain, a combination of these drugs is the accepted worldwide approach in symptomatic SARS-CoV-2 infection.

Beyond their limited evidence for treating COVID-19, the scientific community is aware of adverse effects, in particular the QT interval prolongation. There are sporadic data reporting QT interval prolongation with hydroxychloroquine, when used in patients with systemic lupus erythematosus. On the other hand, azithromycin is a well-recognized ventricular arrhythmia and sudden cardiac death (SCD) aetiology, even in the absence

\footnotetext{
${ }^{1}$ Cardiology Department, Virgen de Las Nieves University Hospital, Avenida de las fuerzas armadas 2, 18014 Granada, Spain. 'Biosanitary Research Institute (IBS), Av. Del conocimiento, 18012 Granada, Spain. ${ }^{3}$ Centro Nacional de Investigaciones Cardiovasculares Carlos III (CNIC), 28029 Madrid, Spain. ${ }^{4}$ Department of Internal Medicine, Virgen de Las Nieves University Hospital, Avenida de las fuerzas armadas 2, 18014 Granada, Spain. ${ }^{5}$ Department of Pneumology, Virgen de Las Nieves University Hospital, Avenida de las fuerzas armadas 2, 18014 Granada, Spain. ${ }^{6}$ Emergency Department, Virgen de Las Nieves University Hospital, Avenida de las fuerzas armadas 2, 18014 Granada, Spain. ${ }^{\circledR}$ email: jimenez.jaimez@gmail.com
} 
of significative QT interval prolongation ${ }^{6-8}$. Finally, lopinavir/ritonavir is listed as a drug with possible risk of torsade de pointes (TdP) at crediblemeds.org.

The effect of the combination of one or more of these drugs on cardiac rhythm or repolarization has been recently analysed in heterogeneous samples of COVID-19 patients. Our aim is to study the QT interval behaviour in a selected population of not critically ill patients with COVID-19 treated with hydroxychloroquine, azithromycin and/or antiviral drugs.

\section{Methods}

Population and study design. An unicentric cross-sectional study was conducted between March 23 and April 24, 2020 in a tertiary referral hospital. Patients presenting to our emergency department (ED) with confirmed diagnosis or high clinical suspicion of SARS-CoV-2 infection and treated whether an inpatient or outpatient management, were identified. Confirmed SARS-CoV-2 infection was defined as positive nasopharyngeal polymerase chain reaction (RT-PCR) and/or positive IgM and IgG serological test. High suspicion diagnosis was determined by the physician criteria based on clinical characteristics, chest imaging, blood test parameters and ruling out common infectious pathogens causing pneumonia, if diagnostic tests were undetermined, negative or non-available. Ambulatory and admitted patients with COVID-19 treated with one or more SARS-Cov-2 experimental drugs (according to our hospital protocol, see appendix I) were included. Patients readmitted for SARS-Cov-2 and those admitted to the Intensive Care Unit (ICU) or with mechanical ventilation at the time of baseline and control ECG were excluded. Clinical protocol was carried out in accordance with relevant guidelines and regulations.

The clinical protocol of the study was approved by the Local Ethics Committee (Virgen de las Nieves Universitary Hospital, Granada). Informed consent was obtained from all the participant patients.

Data collection. Twelve lead ECG was performed in every patient $48 \mathrm{~h}$ after treatment initiation. In addition, most of the patients had a basal ECG collected before the beginning of medical therapy at the ED admission. Electrocardiographic analysis included heart rhythm and rate, P-QRS-T intervals duration, atrioventricular and intraventricular conduction disturbances and ventricular repolarization in all ECGs. Measurement of the QT interval was preferably carried out on lead II, and V5-V6 if needed, starting at the beginning of the Q wave and using the tangent method described by Postema et al. ${ }^{9}$ and corrected with the Bazett's formula. Patients with generalized flat T waves and not reliable ECG recordings were excluded from the analysis. Patients with bundle branch block and paced rhythms were not excluded although QTc interval was assessed by Bazzet and Bogossian formulas. Electrocardiographic assessment was performed by two independent certified cardiac electrophysiologists who were blinded to the patient's information.

Furthermore, baseline investigations included previous comorbidities, chronic treatments, severity illness at ED, complete blood count, coagulation profile, serum biochemical test (creatine kinase, lactate dehydrogenase, electrolytes, renal and liver function), and complementary treatment during hospitalization with QTc interval prolongation risk were also recorded. Additionally, mortality during admission was analysed.

The primary endpoint of the study was to determine the prevalence of clinically significant QT interval prolongation under medical therapy for COVID-19, especially QTc greater than $500 \mathrm{~ms}$ involving a higher arrhythmic risk. Other secondary aims were to detect clinical or analytical independent predictors of QT interval prolongation and quantify the QTc interval increasing from basal ECGs.

Drug therapy. According to our centre protocol, in patients with confirmed or high-suspicion of COVID19 , a combination of two or more of the following agents was proposed: hydroxychloroquine $400 \mathrm{mg}$ BID (loading dose) and $200 \mathrm{mg}$ BID during 5 days, azithromycin $500 \mathrm{mg}$ SID (loading dose) and $250 \mathrm{mg}$ SID during 5 days, and Lopinavir/Ritonavir 400/100 mg BID or Darunavir/Ritonavir 600/100 mg BID during 7 to 14 days at physician criteria based on severity illness.

Statistical analysis. Numerical variables are expressed as mean and standard deviation or, for non-normally distributed variables, as median [interquartile range]. Categorical variables are expressed as absolute and relative frequencies. To assess the agreement between the two observers with respect to both, baseline and control QTc parameter measurements, the Bland-Altman graphical method was used, calculated with the inter$\mathrm{val} \pm 1.96$ times the standard deviation from the mean difference. In addition, the intraclass correlation coefficient was calculated. The Wilcoxon test was used to compare the baseline and control QTc. A bivariate analysis was performed to analyze the variables that are related to the QTc prolongation, establishing $460 \mathrm{~ms}$ as the cut-off point. The categorical variables have been contrasted with the Pearson's chi-square test or Fisher's exact test in cases where applicability conditions were not met. For the quantitative variables, the Student's t-test was used for independent samples and the Mann-Whitney test was used for those variables with a non-normal distribution. With the variables in which a $\mathrm{p}$ value of $<0.20$ was obtained, a multivariate logistic regression model was constructed. For the final model, the odds ratio is calculated along with its $95 \%$ confidence interval. The goodness of fit was compared with the Hosmer-Lemeshow test and its predictive capacity was evaluated with the area under the ROC curve.

Patient and public involvement. There has been no direct public or patient involvement in this research. 


\begin{tabular}{|c|c|c|c|c|}
\hline & Total $(\mathrm{N}=219)$ & $\begin{array}{l}\text { Hydroxychloroquine and/or azithromycin } \\
(\mathrm{N}=105)^{\mathrm{a}}\end{array}$ & $\begin{array}{l}\text { Hydroxychoroquine. azithromycin and } \\
\text { antirretroviral }(\mathrm{N}=114)\end{array}$ & $P$ value \\
\hline \multicolumn{5}{|l|}{ Baseline characteristics no. (\%) } \\
\hline Age, mean (SD)-yr & $63.6(17.4)$ & $67.1(19.3)$ & $60.3(14.7)$ & .002 \\
\hline Male sex & $112(51.1)$ & $46(43.8)$ & $66(57.9)$ & .037 \\
\hline AHT & $109(49.8)$ & $55(52.4)$ & $54(47.4)$ & .459 \\
\hline Structural Heart Disease $^{\mathrm{b}}$ & $33(15.1)$ & $21(20.0)$ & $12(10.5)$ & .050 \\
\hline Obesity & $19(8.7)$ & $8(7.6)$ & $11(9.6)$ & .594 \\
\hline ACEI/ARB & $83(37.9)$ & $39(37.1)$ & $44(38.6)$ & .825 \\
\hline Beta blocker & $39(17.8)$ & $22(21.0)$ & $17(14.9)$ & .243 \\
\hline Antidepressant & $36(16.4)$ & $26(24.8)$ & $10(8.8)$ & .001 \\
\hline \multicolumn{5}{|l|}{ Clinical features no. (\%) } \\
\hline Fever-no. (\%) & $145(66.2)$ & $56(53.3)$ & $89(78.1)$ & $<.001$ \\
\hline Multilobar Pneumonia-no. (\%) & $135(61.6)$ & $47(44.8)$ & 88 (77.2) & $<.001$ \\
\hline Myocarditis & $0(0)$ & $0(0)$ & $(0)$ & - \\
\hline Ferritin-median [IQR], ng/mL & $573.0[205.5-1275.0]$ & $330.0[170.0-514.0]$ & $1040.0[394.3-2215.6]$ & $<.001$ \\
\hline Hs Troponin I, median [IQR], pg/ml & $6.1[2.2-20.0]$ & $11.0[4.0-31.0]$ & $4.7[2.1-10.6]$ & .005 \\
\hline DD-median [IQR], mg/L & $0.9[0.5-2.1]$ & $1.44[0.65-3.9]$ & $0.95[0.6-1.5]$ & .203 \\
\hline LDH-median [IQR], IU/L & $364.0[271.0-458.0]$ & $308.0[268.0-405.0]$ & $391.5[347.5-521.3]$ & $<.001$ \\
\hline CRP-median [IQR], mg/L & $73.0[20.0-156.0]$ & $52.0[22.5-172.0]$ & 84.0 [39.5-177.5] & $<.001$ \\
\hline $\mathrm{K}+-$ mean $(\mathrm{SD}), \mathrm{mEq} / \mathrm{L}$ & $4.0[3.8-4.3]$ & $4.0[3.8-4.4]$ & $4.0[3.8-4.3]$ & .494 \\
\hline $\mathrm{Ca}+2$-mean $(\mathrm{SD}), \mathrm{mg} / \mathrm{dL}$ & $9.2[8.8-9.6]$ & $9.2[8.6-9.6]$ & $9.2[8.9-9.5]$ & .374 \\
\hline Tocilizumab-no. (\%) & $11(5.0)$ & $0(0.0)$ & $11(9.6)$ & .001 \\
\hline Antidepressant-no. (\%) & $27(12.3)$ & $19(18.1)$ & $8(7.0)$ & .013 \\
\hline Neuroleptic-no. (\%) & $19(8.7)$ & $14(13.3)$ & $5(4.4)$ & .019 \\
\hline Exitus-no. $(\%)^{* * \star}$ & $15(8.2)$ & $10(14.5)$ & $5(4.4)$ & .016 \\
\hline
\end{tabular}

Table 1. Baseline and clinical characteristics depending of COVID-19 treatment administered. ACEI, angiotensin converting enzyme inhibitor; AHT, arterial hypertension ARB, angiotensin receptor blocker; CRP, C-reactive protein; DD, D-dimer; ECG, electrocardiogram; Hs, high sensitive; IQR, interquartile range; $\mathrm{LDH}$, lactate dehydrogenase; SD, standard deviation. ${ }^{a}$ Note that $90.5 \%(\mathrm{n}=95)$ of the patients received both drugs, azithromycin and hydroxychloroquine. ${ }^{b}$ Considered: ischemic, hypertensive, dilated, hypertrophic and valvular cardiomyopathy. ${ }^{c}$ Inpatient mortality $(\mathrm{n}=183)$.

\section{Results}

Baseline parameters. Two hundred and nineteen patients with confirmed diagnosis or high suspicion of COVID-19 were included, with a mean age of 63.6 ( \pm 17.4 years, $23-98$ years), 107 were (48.9\%) women. Thirtysix $(16.4 \%)$ were outpatients. Most of the patients $(n=201)$ had a definitive SARS-CoV-2 diagnosis due to a positive RT-PCR or serological test, while the remaining were classified as high suspicion cases basing on chest imaging and clinical course. All patients had a control ECG $48 \mathrm{~h}$ after treatment initiation and 165 patients had a baseline ECG at ED admission. At ED admission, 154 patients (93.3\%) were on sinus rhythm and 11 on atrial fibrillation (6.7\%). One hundred and forty-eight patients $(89.7 \%)$ had a narrow QRS on admission, 3 had left bundle branch block (1.8\%), 9 right bundle branch block (5.5\%), 4 bifascicular block (2.4\%) and 1 ventricular paced rhythm $(0.6 \%)$. Patients were divided in two groups according to the treatment: group 1 (single or double therapy) with 105 patients (47.9\%) who received hydroxychloroquine and/or azithromycin and group 2 (triple therapy) with 114 patients (52.1\%) who received hydroxychloroquine, azithromycin and protease inhibitors (lopinavir-ritonavir or darunavir-ritonavir). Only one patient $(0.5 \%)$ did not received azithromycin and 9 did not received hydroxychloroquine (4.1\%). Baseline characteristics according to treatment are shown in Table 1; patients with triple therapy had a worse clinical presentation during admission, with more fever, more multilobar pneumonia and worse analytics parameters.

QTc interval analysis. Correlation between both ECG observers was excellent basing upon the result of the Blant Altman test (Supplementary Fig. 1) with an intraclass coefficient of 0.903.

The spectrum of QTc distribution after treatment is shown in Fig. 1, with only 4 patients with QTc $>500 \mathrm{~ms}$, most of them cases with wide QRS due to intraventricular conduction disturbances or paced rhythm; one case with narrow QRS complex developed a QTc interval $>500 \mathrm{~ms}$. Only $14.1 \%$ of the cases $(\mathrm{n}=31)$ presented a QTc interval of more than $460 \mathrm{~ms}$ after treatment and no patient presented torsade de pointes or arrhythmic death during admission. Table 2 shows a bivariate model to identify variables associated with the presence of a QTc $>460$ ms. In Fig. 2, statistically significant predictors of QT interval prolongation in the bivariate model are graphically represented. There were no differences in QTc between treatment groups 1 and 2.

The median baseline QTc was $416 \mathrm{~ms}$ (IQR 404-433), and after treatment QTc was prolonged to $423 \mathrm{~ms}$ $(405-438)(\mathrm{P}<0.001)$ (Fig. 3, panel A). An increase in QTc between 0 and $5 \%$ was observed in most of the 


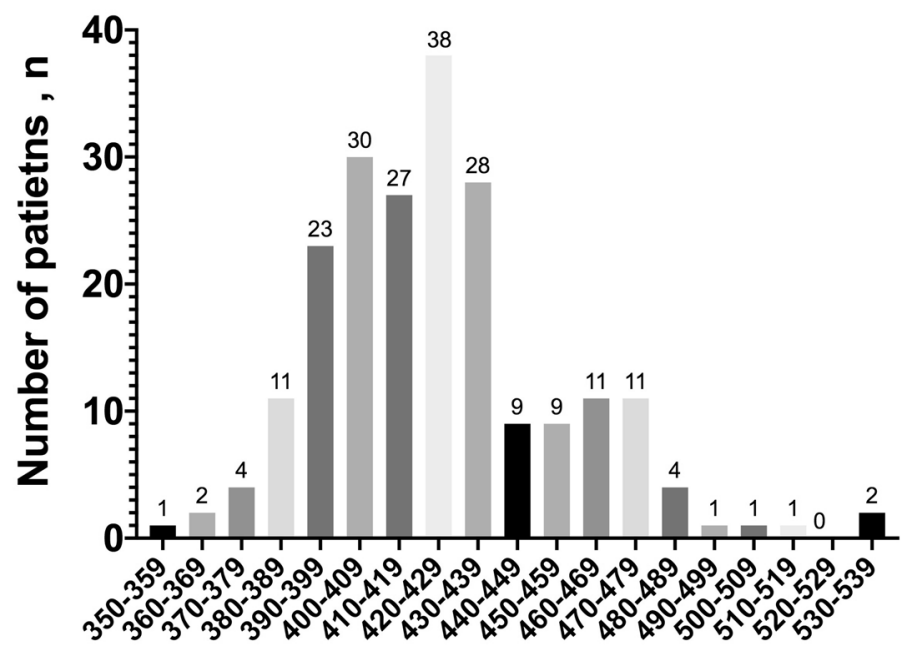

Figure 1. Distribution of patients according to the QTc interval during treatment, at $10 \mathrm{~ms}$ intervals. Only $2.9 \%(n=6)$ of patients presented a QTc of more than $480 \mathrm{~ms}$. Among the 4 patients presenting a QTc interval of $\geq 500 \mathrm{~ms}, 3$ showed a wide QRS due to intraventricular conduction defects (left bundle branch block or right bundle branch block).

patients, with an average increase of $1.8 \%$ (- 1.3 to 5.6\%) (Fig. 3, panel B); importantly, only 10 patients showed an increase greater than $10 \%$. Of note, 9 of them did not presented high-risk markers: a QTc interval $>500 \mathrm{~ms}$, or a QTc variation $>60 \mathrm{~ms}$.

In the multivariate model (Table 3) age was a risk factor, with an OR 1.05 [1009-1095]. For each year of age, the risk of prolonged QTc increased by 5\%. The baseline QTc is a risk factor, with an OR 1.06 [1009-1095]. For each ms of increasing in baseline QTc interval, the risk of prolonged QTc increased by 5\%. Basal potassium acts as a protective factor. For each unit that increases basal potassium, the risk of prolonged QTc is 3.29 times lower (1/0.3039). Predictive ability of the model was very good, with an area under the ROC curve of 0.9130 (supplementary Fig. 2).

Follow-up. Fourteen patients discontinued treatment (6.4\%), in 11 patients (5\%) due to QTc prolongation. The median hospital stay was 7 (5-11) days. After admission, 5 patients required intensive care, and only one of them in needing of orotracheal intubation. Fifteen patients died $(6.8 \%)$ and two of the outpatients required admission during follow-up due to worsening symptoms.

\section{Discussion}

Scientific community is facing against one of the hardest epidemiological crisis in the last century, and safety regarding medical therapies used against SARS-Cov-2 infection seems critical to avoid iatrogenia. Indeed, significant concern about ventricular arrhythmias and SCD in COVID-19 population have emerged, since previous data about QT interval prolongation are reported with the use of hydroxychloroquine and azithromycin, which added to the antiretroviral drugs have been defined as "the corona cocktail" ${ }^{10}$. In this direction, a recent report from the Italian group in Lombardia has warned about the important increase in out-of-hospital cardiac arrests, suggesting a hypothetical link between SARS-CoV-2 infection and this high rate of ventricular arrhythmias ${ }^{11}$. One of the connections might be the QTc prolongation and torsade de pointes induced by drugs (DI-TdP) in ambulatory and admitted, but not ECG-monitored, medically treated patients with COVID-19. Our study focuses on this specific group of patients that, due to a not so serious SARS-CoV-2 disease stage, are not admitted to the ICU, or they even are discharged without admission to hospital. The lack of ECG monitoring would markedly reduce the survival chances of these patients in case of DI-TdP. Our data are strongly reassuring about the use of this combination of drugs in this specific cohort of patients, as we have not detected a clinically relevant QTc interval prolongation attributable to the treatment. Interestingly, our serie is the first, as far as we know, to test the safety of the combination of the three drugs, including antiretrovirals, against COVID-19.

There are some recent reports describing a high percentage of patients developing significant QT interval prolongation (more than $500 \mathrm{~ms}$ ) in patients with COVID-19, albeit in heterogeneous cohort of patients ${ }^{12-14}$. A recent report from the New York group found a significant QTc interval prolongation in a substantial proportion of patients, being more pronounced in patients with congestive heart failure, on amiodarone or with higher basal QTc intervals ${ }^{12}$. They even present a case of DI-Tdp successfully defibrillated. However, the report of TdP cases in COVID-19 patients is scarce and it likely occurs in ECG-monitored patients admitted to the ICU. Strikingly, their median basal QTc interval is quite long, about $440 \mathrm{~ms}$, which is the upper limit for healthy population; something similar was observed in other works with a basal median QTc of $455 \mathrm{~ms}^{13,14}$. This fact suggests the presence of concomitant factors predisposing this extreme QTc interval prolongation response $\mathrm{e}^{14}$. Our data suggest a modest QTc interval prolongation, with just one case, in the absence of LBBB or ventricular paced QRS, greater than $500 \mathrm{~ms}$. 


\begin{tabular}{|c|c|c|c|}
\hline & $\begin{array}{l}\text { QTc } \leq 460 \mathrm{~ms} \\
(\mathrm{n}=188)^{\mathrm{a}}\end{array}$ & $\begin{array}{l}\text { QTc }>460 \mathrm{~ms} \\
(\mathrm{n}=31)^{\mathrm{a}}\end{array}$ & P value \\
\hline \multicolumn{4}{|l|}{ Baseline characteristics no. (\%) } \\
\hline Age-mean (SD), yr & $61.3(17.0)$ & $77.3(2.9)$ & $<.001$ \\
\hline Male sex & $99(52.7)$ & $13(41.9)$ & 0.268 \\
\hline Outpatient management & $35(18.6)$ & $1(3.2)$ & .032 \\
\hline AHT & $87(46.3)$ & $22(71.0)$ & .011 \\
\hline Structural Heart Disease & $20(10.6)$ & $13(41.9)$ & $<.001$ \\
\hline Obesity & $18(9.6)$ & $1(3.2)$ & .487 \\
\hline ACEI/ARB & $66(35.1)$ & $17(54.8)$ & .046 \\
\hline Beta blocker & $31(16.5)$ & $5(25.8)$ & .209 \\
\hline Antidepressant & $24(12.8)$ & $12(38.7)$ & $<.001$ \\
\hline \multicolumn{4}{|l|}{ ECG at baseline-median (IQR) } \\
\hline PR interval, ms & $150[140-160]$ & $150[140-180]$ & 0.915 \\
\hline QRS interval, ms & $90[80-100]$ & $100[90-122]$ & $<.001$ \\
\hline QT interval, ms & $360[330-370]$ & $360[340-400]$ & 0.125 \\
\hline QTc interval, ms & $414[400-427]$ & $446[433-476]$ & $<.001$ \\
\hline Heart Rate, bpm & 81 [72-99] & 94 [75-101] & 0.113 \\
\hline \multicolumn{4}{|l|}{ Clinical features-median (IQR) } \\
\hline Length of stay, d & $7.5[5.0-11.0]$ & $7.0[5.0-9.8]$ & .499 \\
\hline Fever-no. (\%) & $126(67.0)$ & $19(61.3)$ & .532 \\
\hline Multilobar Pneumonia-no. (\%) & $112(59.6)$ & $23(74.2)$ & .121 \\
\hline Ferritin-ng/mL & $571.0[196.8-1337.3]$ & $642.0[258.0-1119.0]$ & .771 \\
\hline Hs Troponin I-pg/ml & $5.0[2.0-13.0]$ & $22.4[9.4-69.0]$ & $<.001$ \\
\hline $\mathrm{DD}-\mathrm{mg} / \mathrm{L}$ & $0.9[0.5-1.9]$ & $1.4[0.8-13.5]$ & .008 \\
\hline LDH-IU/L & $361.5[262.3-457.5]$ & $392.0[301.0-505.0]$ & .175 \\
\hline CRP-mg/L & $67.0[18.1-146.0]$ & $78.0[37.0-209.0]$ & .084 \\
\hline $\mathrm{Na}+-\mathrm{mEq} / \mathrm{L}^{\mathrm{b}}$ & $139.0[136.0-141.0]$ & $138.5[136.3-141.0]$ & .649 \\
\hline $\mathrm{K}+-\mathrm{mEq} / \mathrm{L}^{\mathrm{b}}$ & $4.1[3.8-4.4]$ & $3.7[3.4-4.3]$ & .087 \\
\hline $\mathrm{Ca}+2-\mathrm{mg} / \mathrm{dL}^{\mathrm{b}}$ & $9[8.6-9.4]$ & $8.5[8.3-9.0]$ & .009 \\
\hline Tocilizumab-no. (\%) & $9(4.8)$ & $2(6.5)$ & .658 \\
\hline Antidepressant-no. (\%) & $16(8.5)$ & $11(35.5)$ & $<.001$ \\
\hline Neuroleptic-no. (\%) & $13(6.9)$ & $6(19.4)$ & .035 \\
\hline Treatment discontinuation-no. $(\%)^{\mathrm{c}}$ & $6(3.2)$ & $8(25.8)$ & $<.001$ \\
\hline Exitus-no. (\%) & $10(5.3)$ & $5(16.1)$ & .027 \\
\hline
\end{tabular}

Table 2. Baseline, ECG and clinical characteristics of patients according to the QTc interval at $\geq 48 \mathrm{~h}$ from treatment initiation. ACEI, angiotensin converting enzyme inhibitor; AHT, arterial hypertension ARB, angiotensin receptor blocker; CRP, C-reactive protein; DD, D-dimer; ECG, electrocardiogram; Hs, high sensitive; IQR, interquartile range; $\mathrm{LDH}$, lactate dehydrogenase; $\mathrm{SD}$, standard deviation. ${ }^{\text {Both }}$ groups were created using a QTc cut-off of $460 \mathrm{~ms}$ in the ECG at $\geq 48 \mathrm{~h}$ from treatment initiation. ${ }^{\mathrm{b}}$ Plasma ions were analyzed at the time the control ECG was performed. ${ }^{\mathrm{c}}$ Treatment discontinuation because of findings in the ECG at $\geq 48 \mathrm{~h}$ from treatment initiation.

There may be several reasons for this different observation. First of all, ours is a homogenous cohort of patients excluding severely ill patients in needing of mechanical ventilation nor central nervous system depressants drugs; basal QTc in our serie is similar to the one observed in general population ${ }^{15}$. Previous studies with extremely prolonged basal QT intervals may not reflect the patient's real-world baseline repolarization status. These data have been replicated recently by the group from Yale ${ }^{16}$, that describe a significantly prolonged QTc interval after treatment among patients admitted to ICU after reviewing more than two thousands ECGs of patients with COVID-19; on the other hand, previous studies focus mostly on American population ${ }^{12-15}$, with lack of large series in other regions. A single European work showed a significantly prolonged QT interval in more than $20 \%$ of the sample ${ }^{17}$, but in this case patients were treated with chloroquine, which is known to affect QT interval in a greater degree than HCQ. In fact, chloroquine has even been tested in a randomized clinical trial for patients with COVID-19, showing significant QT interval prolongation, although in higher doses than the conventional used $^{18}$. Another European cohort in a very limited sample of patients, most of them admitted to the ICU and intubated, showed a significant proportion of patients with QTc interval greater than $500 \mathrm{~ms}^{19}$. These data differ with ours, although basal population and treatment approach is different too. These more severe patients are likely to suffer myocardial damage that could predispose to QT interval prolongation, albeit a direct relationship between COVID-19 and QT prolongation due to myocarditis has not been demonstrated. A recent multicentric 


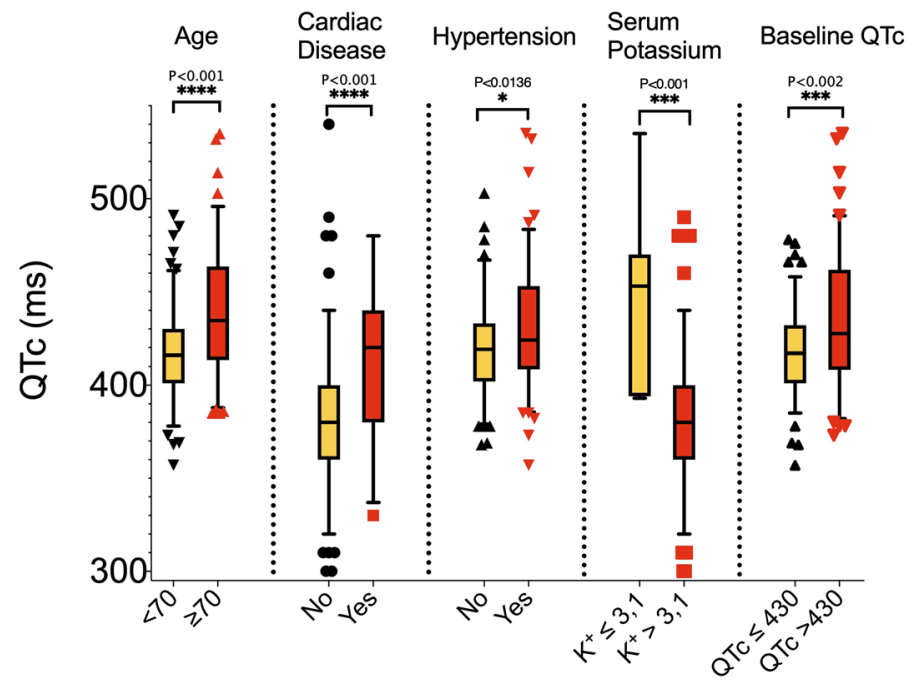

Figure 2. Representation of QTc interval of relevant variables associated with the presence of a QTc $>460 \mathrm{~ms}$ (cardiac disease, age, hypertension and the previous treatment with antidepressants).
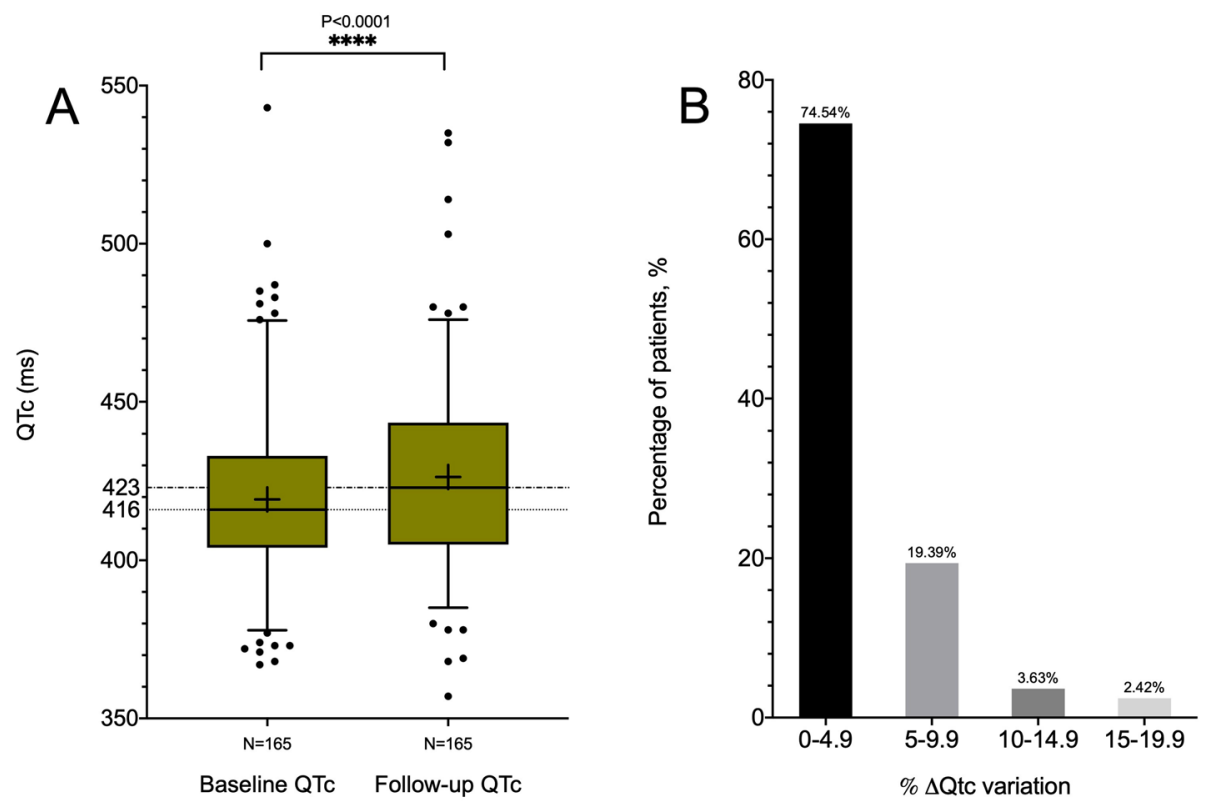

Figure 3. Panel A. Representation of QTc interval at baseline (at treatment initiation) and at follow-up (at least $48 \mathrm{~h}$ on treatment). The median of the baseline QTc was 416 (404-433) ms; after treatment QTc was prolonged to 423 (405-438) ms. Panel B. Distribution of patients according to the percentage increase of the QT interval with respect to the basal. The vast majority of patients presented a mild (0-9.9\%) raise in QTc during treatment.

\begin{tabular}{|l|r|l|l|l|}
\hline & \multirow{2}{*}{ Sig } & \multirow{2}{*}{ OR } & \multicolumn{2}{|l|}{ OR 95\% CI } \\
\cline { 4 - 6 } & .0015 & 1.051 & Lower & Upper \\
\hline Age & .043 & 0.30 & 0.095 & 1.094 \\
\hline K + at baseline & $<.001$ & 1.061 & 1.032 & 1.091 \\
\hline QT interval at baseline & &
\end{tabular}

Table 3. Multivariate analysis. CI, confidence interval; OR, odds ratio; Sig, statistical significance. 
registry has shown an increased risk of arrhythmic risk in patients taking antimalarial + /- macrolide, but with no clear relation with an underlying QT interval prolongation ${ }^{20}$.

It is well recognized that genetic background is the key point to develop QTc interval prolongation and DI-TdP. Some genetic polymorphisms are common in specific geographical areas and may predispose, under environmental circumstances such as drugs or electrolyte disturbances, to prolong the QT interval. Particularly, some polymorphisms in SCN5A are present in a high proportion of American population of African-descent, such as p.Ser1103Tyr; this variant is absent in Europeans and has been proven to increase the late/persistent sodium current, with a basal modest proarrhyhtmic effect, but an enhanced risk of DI-TdP under clinical scenarios that reduce the repolarization reserve such as hypoxia, acidosis or adverse clinical status with advanced age, ${ }^{10,21-27}$. This is something we have also observed in our serie, as advanced age was an independent predictor of QT interval prolongation. However, in the absence of a proarrhythmic genetic variant, these unfavourable conditions seem to be not enough to damage the repolarization reserve and are not able to create the perfect substrate for the appearance of ventricular arrhythmias and SCD. This fact might be contributing to the observed difference between our serie and the ones by the Americans.

Taking our data into consideration, our hypothesis to explain the higher rate of out of hospital cardiac arrests described by some groups ${ }^{11}$ does not support the DI-TdP etiology. Recent studies have shown a worrisome decline in the number of patients treated with primary coronary intervention for acute coronary syndrome during the pandemia ${ }^{28,29}$. Fear of patients to be admitted to health centers as well as the strict public health laws may have contributed to this phenomenon, in a higher grade than the sporadic cases of DI-TdP in patients with COVID-19.

\section{Conclusions}

Ambulatory and not critically ill patients with COVID-19 treated with hydroxychloroquine, azithromycin and/ or antiretrovirals develop a significant, but not relevant, QT interval prolongation. Advanced age, prolonged baseline QTc and low basal potassium levels are independent markers for this prolongation. Although caution is needed to identify basal risk predictors, these data are reassuring for this specific population.

Limitations. First, due to the centre's treatment protocol all patients received the medication and a control group of untreated positive COVID-19 patients was not available. Second, the absence of baseline ECG in some patients and limited follow-up may underestimate the real incidence of high risk QTc prolongation.. Finally, considering the daily update on the disease, we may have not reflected on some relevant clinical variables that could have confounded clinical conclusions.

\section{Data availability}

The datasets generated during and/or analyzed during the current study are available from the corresponding autor on reasonable request.

Received: 7 August 2020; Accepted: 23 November 2020

Published online: 08 December 2020

\section{References}

1. Huang, C. et al. Clinical features of patients infected with 2019 novel coronavirus in Wuhan China. Lancet 395, 497-506. https:// doi.org/10.1016/S0140-6736(20)30183-5 (2020).

2. Zhou, F. et al. Clinical course and risk factors for mortality of adult inpatients with COVID-19 in Wuhan, China: a retrospective cohort study. Lancet 395, 1054-1062. https://doi.org/10.1016/S01406736(20)30566-3 (2020).

3. Giudicessi, J. R., Noseworthy, P. A., Friedman, P. A., Ackerman, M. J. Urgent guidance for navigating and circumventing the QTc prolonging and torsadogenic potential of possible pharmacotherapies for COVID-19. Mayo Clin. Proc. https://doi.org/10.1016/j. mayocp.2020.03.024.

4. Gautret, P. et al. Hydroxychloroquine and azithromycin as a treatment of COVID-19: results of an open-label non-randomized clinical trial. Int. J. Antimicrob. Agents. https://doi.org/10.1016/j.ijantimicag.2020.105949 (2020).

5. Groneberg, D. A. et al. Treatment and vaccines for severe acute respiratory syndrome. Lancet Infect. Dis. 5, 147-155. https://doi. org/10.1016/s1473-3099(05)01307-1 (2005).

6. Roden, D. M., Harrington, R. A. \& Poppas, A. AMC Russo for drug interactions on QTc in exploratory COVID-19 (Coronavirus Disease 2019 treatment. Circulation https://doi.org/10.1161/CIRCULATIONAHA.120.047521 (2020).

7. Zhang, M. et al. Electrophysiologic studies on the risks and potential mechanism underlying the proarrhythmic nature of azithromycin. Cardiovasc. Toxicol. 17, 434-440 (2017).

8. Capel, R. A. et al. Hydroxychloroquine reduces heart rate by modulating the hyperpolarization-activated current If: novel electrophysiological insights and therapeutic potential. Heart Rhythm. 12, 2186-2194 (2015).

9. Postema, P. \& Wilde, A. The measurement of the QT interval. Curr. Cardiol. Rev. 10(3), 287-294. https://doi.org/10.2174/15734 03X10666140514103612 (2014).

10. Giudicessi, J. R., Roden, D. M., Wilde, A. A. M. \& Ackerman, M. J. Genetic susceptibility for COVID-19-associated sudden cardiac death in african americans. Heart Rhythm https://doi.org/10.1016/j.hrthm.2020.04.045 (2020).

11. Baldi, E. et al. Out-of-hospital cardiac arrest during the Covid-19 outbreak in Italy. N. Engl. J. Med. https://doi.org/10.1056/NEJMc 2010418 (2020).

12. Chorin, E. et al. QT Interval prolongation and torsade de pointes in patients with COVID-19 treated with hydroxychloroquine/ azithromycin. Heart Rhythm. https://doi.org/10.1016/j.hrthm.2020.05.014 (2020).

13. Mercuro, N. J. Risk of QT interval prolongation associated with use of hydroxychloroquine with or without concomitant azithromycin among hospitalized patients testing positive for Coronavirus Disease, et al 2019 COVID-19. JAMA Cardiol. https://doi. org/10.1001/jamacardio.2020.1834 (2020).

14. Saleh, M. et al. The effect of chloroquine, hydroxychloroquine and azithromycin on the corrected QT interval in patients with SARS-CoV-2 infection. Circ. Arrhythm. Electrophysiol. https://doi.org/10.1161/CIRCEP.120.008662 (2020).

15. Goldenberg, I., Moss, A. J. \& Zareba, W. QT interval: how to measure it and what is "normal". J. Cardiovasc. Electrophysiol. 17, 333-336 (2006).

16. Jain, S. et al. Enhanced ECG monitoring of COVID-19 patients. Heart Rhythm. https://doi.org/10.1016/j.hrthm.2020.04.047 (2020). 
17. van den Broek, M. P. H. et al. Chloroquine-induced QTc prolongation in COVID-19 patients. Neth. Heart J. https://doi.org/10.1007/ s12471-020-01429-7 (2020).

18. Borba, M. G. S. et al. Effect of high vs low doses of chloroquine diphosphate as adjunctive therapy for patients hospitalized with severe acute respiratory syndrome coronavirus 2 (SARS-CoV-2) infection: a randomized clinical trial. JAMA Netw. Open 3, e208857 (2020).

19. Bessière, F., et al. Assessment of QT intervals in a case series of patients with Coronavirus Disease 2019 (COVID-19) infection treated with hydroxychloroquine alone or in combination with azithromycin in an intensive care unit. JAMA Cardiol. https://doi. org/10.1001/jamacardio.2020.1787 (2020).

20. Mandeep, R. M., Sapan, S. D. \& Amit, N. P. Hydroxychloroquine or chloroquine with or without a macrolide for treatment of COVID-19: a multinational registry analysis. Lancet https://doi.org/10.1016/S0140-6736(20)31180-6 (2020).

21. Giudicessi, J. R., Roden, D. M., Wilde, A. A. M. \& Ackerman, M. J. Classification and reporting of potentially proarrhythmic common genetic variation in long QT syndrome genetic testing. Circulation 137, 619-630 (2018).

22. Burke, A. et al. Role of SCN5A Y1102 polymorphism in sudden cardiac death in blacks. Circulation 112, 798-802 (2005).

23. Sun, A. Y. et al. The S1103Y cardiac sodium channel variant is associated with implantable cardioverter-defibrillator events in blacks with heart failure and reduced ejection fraction. Circ. Cardiovasc. Genet. 4, 163-168 (2011).

24. Plant, L. D. et al. A common cardiac sodium channel variant associated with sudden infant death in African Americans, SCN5A S1103Y. J. Clin. Invest. 116, 430-435 (2006).

25. Splawski, I. et al. Variant of SCN5A sodium channel implicated in risk of cardiac arrhythmia. Science 297, 1333-1336 (2002).

26. Akylbekova, E. L. et al. Gene-environment interaction between SCN5A-1103Y and hypokalemia influences QT interval prolongation in African Americans: the Jackson Heart Study. Am. Heart J. 167, 116.e111-122.e111 (2014).

27. Van Norstrand, D. W., Tester, D. J. \& Ackerman, M. J. Overrepresentation of the proarrhythmic, sudden death predisposing sodium channel polymorphism S1103Y in a population-based cohort of African-American sudden infant death syndrome. Heart Rhythm 5, 712-715 (2008).

28. Garcia, S. et al. Journal pre-proof reduction in ST-segment elevation cardiac catheterization laboratory activations in the United States during COVID-19 Pandemic. J. Am. Coll. Cardiol. https://doi.org/10.1016/j.jacc.2020.04.011 (2020).

29. Metzler, B., Siostrzonek, P., Binder, R. K., Bauer, A. \& Reinstadler, S. J. Decline of acute coronary syndrome admissions in Austria since the outbreak of COVID-19: the pandemic response causes cardiac collateral damage. Eur. Heart J. 41, 1852-1853. https:// doi.org/10.1093/eurheartj/ehaa314 (2020).

\section{Acknowledgements}

We are grateful to our colleagues who contributed collecting the ECGs from the COVID-19 patients, in such a difficult context, as well to nurses and auxiliary and administrative personal that have taken part in this project in different ways. Particularly thankful we are to Manuel Molina, Pablo Sánchez Millán, Juan Emilio Alcalá López, Laura Pertejo Manzano, Vicente Alcalde Martínez, Ricardo Rivera López, Joaquín Sánchez Gila, Guillermo Gutiérrez Ballesteros, Lorena González Camacho, Emilio Constan de la Revilla, Alicia Almagro Romero, Eva Cabrera Borrego, Lucía Torres Quintero, Rocío Parrilla Linares, Miguel Morales García, Jesús Peña, Antonio Arriaga, Jose Sánchez Moreno, Isabel Merino, Jesús López Muñoz, Elena Sola García, Nuria Navarrete, José Manuel Díaz López, Ana Pardo and Laura Lamarca and Rosario García García and Juan Jiménez Alonso.

\section{Author contributions}

All the authors has contributed to the planning, conduct, or reporting of the work described in the article; J.J.J. is responsible for the overall content as guarantor(s).

\section{Competing interests}

The authors declare no competing interests.

\section{Additional information}

Supplementary information is available for this paper at https://doi.org/10.1038/s41598-020-78360-9.

Correspondence and requests for materials should be addressed to J.J.-J.

Reprints and permissions information is available at www.nature.com/reprints.

Publisher's note Springer Nature remains neutral with regard to jurisdictional claims in published maps and institutional affiliations.

(c) (i) Open Access This article is licensed under a Creative Commons Attribution 4.0 International License, which permits use, sharing, adaptation, distribution and reproduction in any medium or format, as long as you give appropriate credit to the original author(s) and the source, provide a link to the Creative Commons licence, and indicate if changes were made. The images or other third party material in this article are included in the article's Creative Commons licence, unless indicated otherwise in a credit line to the material. If material is not included in the article's Creative Commons licence and your intended use is not permitted by statutory regulation or exceeds the permitted use, you will need to obtain permission directly from the copyright holder. To view a copy of this licence, visit http://creativecommons.org/licenses/by/4.0/.

(C) The Author(s) 2020 